\title{
Body Height and Its Estimation Utilizing Arm Span Measurements in Adolescents From Northern Region in Montenegro
}

\author{
Marija Bubanja'1 \\ 'University of Montenegro, Faculty for Sport and Physical Education, Niksic, Montenegro
}

\begin{abstract}
The purpose of this research was to examine Montenegrin adolescents' body height from the northern region. Also, the research examines an arm span as an alternative assessment for measuring the body height which varies from region to region in Montenegro. The research surveyed 345 adolescents (164 males and 181 females) from the northern region of Montenegro. Anthropometric measurements were performed according to the protocol of the International Society for the Advancement of Kinanthropometry (ISAK). The final outcome of the research are mean values and standard deviations related to anthropometric measurements. The relationship between the body height and the arm span was determined by using simple correlation coefficients and their $95 \%$ confidence interval. This was followed by a linear regression analysis which was utilized to determine to what extent the arm span can reliably predict the body height. The results show that adolescents from the north part of Montenegro are high; men have an average body height of $182.89 \pm 6.39$ centimeters and the arm span of $182.95 \pm 7.04$ centimeters, while the average height of the female population is $169.47 \pm 6.08$ centimeters with a arm span of $168.02 \pm 7.40$ centimeters. Compared to other studies, the results of this research have shown that this population is taller than most nations throughout Europe. Moreover, as it was expected, the arm span can predict reliably the body height when it comes to this population.
\end{abstract}

Key words: Body Height, Arm Span, Anthropometry, Adolescents, Montenegro

\section{Uvod}

U naučnoj literaturi dobro je poznato da je mjerenje tjelesne visine značajno u mnogim situacijama (Arifi i sar., 2017; Gardašević, Rasidagić, Krivokapić, Ćorluka i Bjelica, 2017; Bjelica, Popović, Kezunović, Petković, Jurak i Grasgruber, 2012; Popović, Bjelica, Tanase i Milasinović, 2015; Popovic, 2017): to je relativna mjera veličine tijela i daje procjenu nutritivnog statusa, te značajnu mjeru utvrđivanja osnovnih energetskih zahtjeva, standardizaciju mjera fizičke sposobnosti, procjene rasta djeteta, predviđanja i standardizacije fizioloških varijabli i identifikacije talenata itd. (Golshan, Amra i Hoghoghi, 2003; Mohanty, Babu i Nair, 2001; Ter Goon, Toriola, Musa i Akusu, 2011; Popović, Bjelica, Molnar, Jaksić i Akpinar, 2013; Popović, Bjelica i Had- zić, 2014; Popovic, Arifi, Bjelica, 2017a; Popovic, i Bjelica, 2016; Popovic, i Bjelica, 2017; Popovic, Bjelica, Georgijev, Krivokapic, Milasinovic, 2016). Odnos dugih kostiju i visine tijela varira u različitim etničkim i rasnim grupama, kao i različitim regionima (Arifi, 2017; Milasinović, Popović, Matić, Gardasević, i Bjelica, 2016; Milasinović, Popović, Jaksić, Vasiljević, i Bjelica, 2016; Gardasevic, 2018; Gardasevic, 2018a; Gardasevic, Masanovic, i Arifi, 2018; Gardasevic, Masanovic, i Arifi, 2018a; Gardasevic, Masanovic, i Arifi, 2018b; Mašanović, 2017; Masanovic, 2018; Masanovic, 2018a; Masanovic, 2018b; Masanović, Gardasević, i Arifi, 2018c; Masanović, Gardasević, i Arifi, 2018d; Masanović, Gardasević, i Arifi, 2018e; Masanović, Gardasević, i Arifi, 2018f; Masanović, Gardasević, i Arifi, 2018g; Popovic, Gardasevic, Ma-

Correspondence:

Montenegro M.Bubanja

Gport University of Montenegro, Faculty for Sport and Physical Education, Narodne omladine bb, 81400 Niksic, Montenegro E-Mail: marijabubanja@ymail.com 
sanovic, Arifi, Bjelica, 2017b; Popovic, Bjelica, 2016). Visoki ljudi mogu imati veću dugovječnost, te imati manji rizik kardiovaskularnih i respiratornih bolesti, ali mogu imati veći rizik od nekih vrsta karcinoma (Quanjer i saradnici, 2014). Veća tjelesna visina takođe može biti važan faktor od kojeg zavisi uspjeh nekih sportista u raznim sportovima (Popović, Bjelica, Petković i Muratović, 2012; Popović, Bjelica, Jaksić i Hadzić, 2014b). Neuobičajna visina crnogorskih gorštaka bila je činjenica koju su evropski antropolozi prepoznali prije više od 100 godina (Bjelica, Popović, Kezunović, Petković, Jurak i Grasgruber, 2012). Pineau i sar. (2005) doprinijeli su ažuriranju prosječnih visina tijela među evropskim populacijama. Oni su u svom istraživanju dokazali da je, suprotno opštem vjerovanju, žensko stanovništvo u Dinarskim Alpima, sa prosječnom visinom od 171 centimetra, priblizno drugoj po visini tijela populaciji u Holandiji, dok je muška populacija Dinarskih Alpa u prosjeku najviša u Evropi. Uzorak od 800 crnogorskih muškaraca koje je izmjerio Robert W. Ehrich (Coon, 1975) početkom 20. vijeka davao je najveći prosjek u cijeloj Evropi 177 cm, dok su se neke oblasti približavale 178 centimetara. Stoga je ova studija izazvala mnoge naučnike da vjeruju da bi crnogorska populacija mogla biti najviša populacija u Evropi. Međutim, Bjelica i njegovi saradnici (2012) potvrđuju da je ženska populacija visoka, ali ne i najviša sa 168,37 centimetara, dok je muška populacija takođe visoka, ali ne i najviša sa 183,2 centimetra. Novije istraživanje sprovedeno kao nacionalno istraživanje (Popović, 2016; Popović, Bjelica i Hadžić, 2014; Popovic, Milasinovic, Matic, Gardasevic, Bjelica, 2016; Popovic, Milasinovic, Jaksic, Vasiljevic, Bjelica, 2016; Popovic Bjelica, Milasinovic, Gardasevic, 2016; Popovic, Bjelica, Milasinovic, Gardasevic, Rasidagic, 2016;) gdje su uzorak ispitanika predstavljali učenici završne godine srednje škole, potvrdili su rezultate prethodne studije i ustanovili da je prosječna visina muške populacije u Crnoj Gori bila 183.74 centimetra. Autori su vjerovali da je populacija koja živi u sjevernoj regiji možda viša od prosječnih Dinarskih Alpa i viša od stanovništva iz centralne i južne regije (Bubanja, Vujović, Tanase, Hadžić i Milašinović, 2015; Milašinović, Popović, Jakšić, Vasiljević i Bjelica, 2016a; Milašinović, Gardašević, Bjelica, 2017) uglavnom zbog pretpostavke specifičnih fizičkih struktura karakterističnih za ljude iz sjevernog dijela Crne Gore.

Vođeni pretpostavkom da su tjelesna visina i raspon ruku međusobno povezani, a potkrijepljeni podacima dobijenim iz istraživanja središnje crnogorske regije (Vujović i sar., 2015) i južnih regija (Milasinović, Popović, Matić, Gardasević i Bjelica, 2016a; Milasinović, Popović, Jakšić, Vasiljević i Bjelica, 2016b), u ovom radu će se istražiti visina tijela, kao i odnos visine i raspona ruku u sjevernoj regiji. S obzirom da postoji razlika u odnosu na središnju i južnu regiju zbog višestrukih komponenti, kao što su: životni uslovi, klima, konfiguracija terena, itd. Svrha ovog istraživanja je ispitati visinu tijela kod crnogorskih adoloscenata iz sjeverne regije, kao i odnos između visine tijela i raspona ruku, koja bi varirala od regije do regije u Crnoj Gori.

\section{Metod}

Obim ove studije kvalifikuje 345 adoloscenata (164 muškaraca, 181 djevojaka) završne godine srednje škole iz sjeverne regije u Crnoj Gori. Ova grupa je izabrana zato što rast pojedinca prestaje u ovom uzrastu i nema gubitka tjelesne težine. Prosječna starost ispitanika bila je $17.96 \pm 0.44$ godine (raspon od 17 do 19 godina).

Prema Marfell-Jones, Olds, Stewart, i Carter (2006), antropometrijska mjerenja, uključujući visinu tijela i raspon ruku, uzeti su u skladu sa protokolom Međunarodnog društva za unapređenje kinantropometrije (ISAK). Starost pojedinaca određena je direktno iz njihovog prijavljenog datuma rođenja.

Analiza je izvršena korišćenjem statističkog paketa za društvene nauke (SPSS) verzija 20.0. Aritmetička sredina i stadardna devijacija (SD) su izračunate za obije antropometrijske varijable. Odnos između visine tijela i raspona ruku utvrđen je korištenjem koeficijenta korelacije i njihovog $95 \%$ intervala pouzdanosti. Zatim je izvršena linearna regresiona analiza kako bi se utvrdilo u kojoj mjeri raspon ruku može pouzdano predvidjeti visinu tijela, sa statističkom značajnošću od $\mathrm{p}<0.05$.

\section{Rezultati}

Rezultati antropometrijskih mjerenja za oba pola prikazani su u tabeli 1 . Prosječna visina tijela za muškarce je 182.89 \pm 6.39 centimetara, rangirana sa minimalnim i maksimlnim rezultatima od 163.0 - 198.0 centimetara. Za djevojke prosjek visine tijela iznosi $169.47 \pm 6.08$ centimetara, rangiran sa minimalnim i maksimalnim rezultatom od 155.5 - 185.6 centimetara. Prosječna vrijednost raspona ruku za muškarce je $182.95 \pm 7.04$ centimetara, sa minimalnim i maksimalnim rezultatom od 160.0 - 201.5 centimetara, dok je prosječna vrijednost raspona ruku kod djevojaka bila $168.02 \pm 7.40$ centimetara, rangirana sa minimalnim i maksimalnim rezultatom od 147.8 - 188.3 centimetara.

Tabela 1. Antropometrijski parametri adoloscenata

\begin{tabular}{ccc}
\hline \multirow{2}{*}{ Pol } & $\begin{array}{c}\text { Visina Tijela } \\
(\text { Mean } \pm \text { SD) }\end{array}$ & $\begin{array}{c}\text { Raspon Ruku } \\
\text { (Mean } \pm \text { SD) }\end{array}$ \\
\hline \multirow{2}{*}{ Muški } & $163.0-198.0$ & $160.0-201.5$ \\
& $(182.89 \pm 6.39)$ & $(182.95 \pm 7.04)$ \\
\multirow{2}{*}{ Ženski } & $155.5-185.6$ & $147.8-188.3$ \\
& $(169.47 \pm 6.08)$ & $(168.02 \pm 7.40)$ \\
\hline
\end{tabular}

Koeficijenti korelacije i njihova 95\%-na analiza intervala pouzdanosti između antropometrijskih mjerenja prikazani su u tabeli 2. Odnos između visine tijela i raspona ruku za oba pola bio je signifikantan $(\mathrm{p}<0.000)$, s koeficijentima korelacije (muškarci 0.820, djevojke 0.840).

Tabela 2. Korelacija između visine tijela i raspona ruku

\begin{tabular}{cccc}
\hline Pol & $\begin{array}{c}\text { Koeficijent } \\
\text { Korelecije }\end{array}$ & $\begin{array}{c}\text { 95\% Interval } \\
\text { Pouzdanosti }\end{array}$ & Sig. \\
\hline Muški & 0.820 & $0.771-0.870$ & $<0.000$ \\
Ženski & 0.840 & $0.802-0.878$ & $<0.000$ \\
\hline
\end{tabular}


U tabeli 3. prikazani su rezultati linearne regresije u kojima vidimo visoke vrijednosti koeficijenta regresije, što ukazuje na pozitivan odnos (muškarci 0.820, djevojke 0.840) koji dalje ukazuje na to da dužina raspona ruku značajno predviđa visinu tijela kod crnogorske populacije iz sjeverne regije (muškarci $t=18.267, p<0.000$; djevojke $t=20.719, p<0.000)$, što potvrđuje koeficijent determinacije za muškarce 0.673 i dje- vojke 0.706 . Prvi od svih modela izveden je uključivanjem starosti kao kovarijate. Međutim, utvrđeno je da je doprinos starosti bio beznačajan i stoga je starosna dob bila odbačena, a procjena izvedena kao univarijatna analiza. Visoke vrijednosti regresionog koeficijenta znače da raspon ruku značajno predviđa visinu tijela kod oba pola crnogorskih adolescenata.

Tabela 3. Rezultati linearne regresione analize

\begin{tabular}{cccccc}
\hline Pol & $\begin{array}{c}\text { Regresioni } \\
\text { Koeficijent }\end{array}$ & $\begin{array}{c}\text { Standardna } \\
\text { Greška (SE) }\end{array}$ & $\begin{array}{c}\text { Koeficijent } \\
\text { Determinacije (\%) }\end{array}$ & t-value & Sig. \\
\hline Muški & 0.820 & 4.042 & 0.673 & 18.267 & 0.000 \\
Ženski & 0.840 & 4.028 & 0.706 & 20.719 & 0.000 \\
\hline
\end{tabular}

Odnos između mjerenja raspona ruku i visine tijela među gore navedenim modelima je prikazan kao dijagram rasprši-

$\operatorname{vanja~(Slika~1).~}$

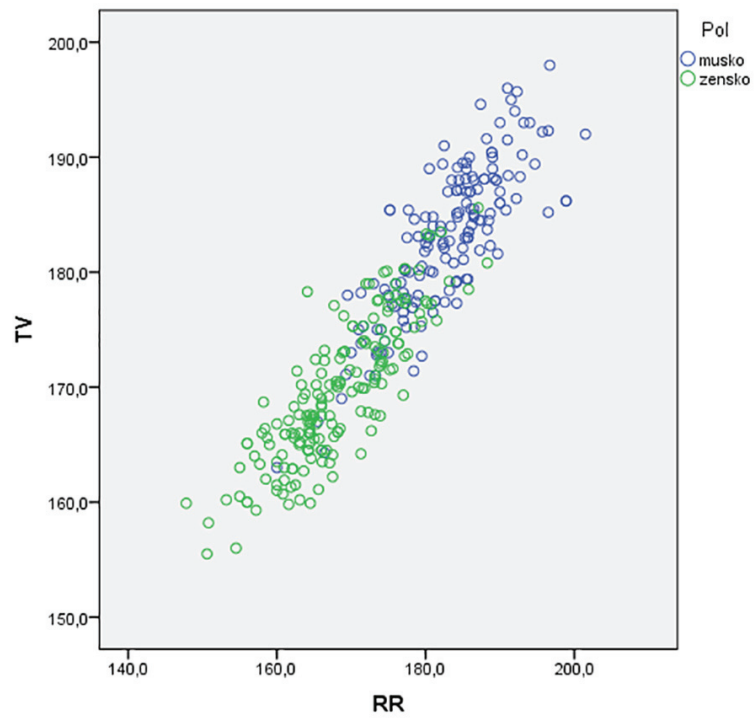

Slika 1. Dijagram raspršivanja i odnos između raspona ruku i visine tijela

\section{Diskusija}

Rezultati ove studije pokazali su da su adolescenti iz sjeverne regije Crne Gore vrlo visoki, u prosjeku 182.89 centimetara muškarci i 169.47 centimetara djevojke. To je nešto manji prosjek od opšteg muškog stanovništva sa 183.21 centimetara i nešto veći od opšteg ženskog stanovništva sa 168.37 centimetara u Crnoj Gori (Bjelica i sar., 2012). Na osnovu rezultata ove studije može se reći da je žensko stanovništvo najveće u Crnoj Gori, što je zaključeno poređenjem sa rezultatima prosječne tjelesne visine iz centralne regije sa 169.24 centimetara (Bubanja i sar., 2015) i južne regije Crne Gore sa 168.73 centimetara (Milašinović i sar., 2016). Prosječna visina muške populacije iz sjeverne regije Crne Gore je manja od populacije iz centralne regije 183.24 centimetra (Vujović i sar., 2015). Međutim, postoji hipoteza da crnogorsko stanovnštvo još uvijek nije ostvarilo svoj puni genetski potencijal jer su u poslednjih nekoliko decenija bili pod uticajem različitih faktora životne sredine kao što su ratovi, loša ekonomska situacija itd. (Popović i sar., 2013). Dakle, autori smatraju da su te okolnosti imale negativan uticaj na sekularni trend u sjevernoj Crnoj Gori, dok se očekuje da će sekularne promjene koje utiču na visinu tijela u narednih 20 godina porasti, upoređujući ih sa razvijenim zemljama u kojima je ovaj trend već završen, kao što je Holandija (Schonbeck i sar., 2013).

S druge strane, očekivano, raspon ruku pouzdano pred- viđa visinu tijela, što se može vidjeti na osnovu postignutih rezultata dobijenih pomoću linearne regresione analize gdje je statistička značajnost bila $\mathrm{p}<0.00$. Odnos između visine tijela i raspona ruku provjeren je pomoću Pearsonove korelacijske analize sa pouzdanošću od $95 \%$ kod muškaraca i djevojaka koji su imali vrlo visoku vrijednost (0.820 i 0.840$)$ međusobnih korelacija. Ovo upućuje na potrebu ispitivanja tjelesne visine kod ostalih regija u Crnoj Gori koje se graniče sa susjednim zemaljama kako bi se konsolidiralo studijsko područje Dinarskih Alpa.

\section{Acknowledgements}

There are no acknowledgements.

\section{Conflict of Interest}

The authors declare that there are no conflict of interest.

Received: 28 April 2018| Accepted: 12 June 2018| Published: 13 July 2018

\section{References}

Arifi, F. (2017). Stature and its estimation utilizing arm span measurements of both gender adolescents from southern region in Kosovo. Sport Science, 10(1), 92-5.

Arifi, F., Bjelica, D., Sermaxhaj, S., Gardasevic, J., Kezunovic, M., \& Popovic, S. (2017a). Stature and its Estimation Utilizing Arm Span Measurements in Kosovan Adults: National Survey. International Journal of Morphology, 35(3):1161-7. 
Bjelica, D., Popović, S., Kezunović, M., Petković, J., Jurak, G., \& Grasgruber P. (2012). Body Height and Its Estimation Utilizing Arm Span Measurements in Montenegrin Adults. Anthropological Notebooks, 18(2), 69-83.

Bubanja, M., Vujovic, D., Tanase, G. D., Hadzic, R., \& Milasinovic, R. (2015) Body Height and Its Estimation Utilizing Arm Span Measurements in Female Adolescents from Central Region in Montenegro. Sport Mont 12(43-45), 277-82

Coon, C. (1975). The races of Europe. Westport, Conn: Greenwood Press.

Gardasevic, J. (2018). Standing Height/Tibia Length Ration in Western-Kosovan Adolescents. Retrieved from SSRN's eLibrary: https://ssrn.com/ abstract $=3138101$

Gardasevic, J. (2018a). Standing Height/Sitting Height Ration in Eastern-Kosovan Adolescents. Retrieved from SSRN's eLibrary: https://ssrn.com/ abstract=3141566Golshan, M., Amra, B., \& Hoghogi, M. A. (2003). Is arm span an accurate measure of height to predict pulmonary function parameters? Monaldi Arch. Chest, Disease 59 (3), 189-92.

Gardasevic, J., Masanovic, B., Arifi, F. (2018). Relationship Tibia Length/Standing Height in Central-Kosovan Adolescents. Retrieved from SSRN's eLibrary: https://ssrn.com/abstract=3138122

Gardasevic, J., Masanovic, B., Arifi, F. (2018a). Relationship Tibia Length/Standing Height in Northern-Kosovan Adolescents. Retrieved from SSRN's eLibrary: https://ssrn.com/abstract=3138112

Gardasevic, J., Masanovic, B., Arifi, F. (2018b). Relationship Tibia Length/Standing Height in Southern-Kosovan Adolescents. Retrieved from SSRN's eLibrary: https://ssrn.com/abstract=3138105

Gardasevic, J., Rasidagic, F., Krivokapic, D., Corluka, M., \& Bjelica, D. (2017) Stature and Its Estimation Utilizing Arm Span Measurements in Male Adolescents from Herzeg-Bosnia Entity in Bosnia and Herzegovina. Montenegrin Journal of Sports Science and Medicine, 6(1), 37-44.

Golhan, M., Amra, B., Hoghoghi, M.A. (2003). Is arm span an accurate measure of height to predict pulmonary function parameters? Monaldi Arch. Chest Dis., 59(3), 189-92.

Marfell-Jones, M., Olds, T., Stewart, A., \& Carter, L. (2006). International standards for anthropometric assessment. Potchesfstroom: Arm span as an independent predictor of pulmonary function parameters: validation and reference values.

Masanovic, B. (2017). Relationship between arm span measurements and body height in Dinaric Alpes population: A systematic review. Journal of Anthropology of Sport and Physical Education, 1(1), 33-37.

Masanovic, B. (2018). Standing Height and its Estimation Utilizing Arm Spam and Foot Length Measurements in Dinaric Alps Population: A Systematic Review. Sport Mont, 16(2), 1-6.

Masanovic, B. (2018a). Standing Height/Sitting Height Relationhip in Western Region in Kosovo. Retrieved from SSRN's eLibrary: https://ssrn. com/abstract $=3138518$

Masanovic, B. (2018b). Tibia Length and Standing Heigh Relationshipt in Eastern Region of Kosovo. Retrieved from SSRN's eLibrary: https://ssrn. com/abstract $=3143118$

Masanovic, B., Gardasevic, J., \& Arifi, F. (2018a). Relationship between foo length measurements and body height: $A$ prospective regional study among adolescents in eastern region of Kosovo. Sport Mont, 16(1), 9-13. doi: $10.26773 /$ smj.180202

Masanovic, B, Gardasevic, J. \& Arifi, F. (2018b). Relationship between Foo Length Measurements and Body Height: A Prospective Regional Study among Adolescents in Northern Region of Kosovo. Anthropologie-International Journal of Human Diversity and Evolution, in pres, https:// doi.org/10.26720/anthro.18.01.23.1.

Masanovic, B., Gardasevic, J., \& Arifi, F. (2018c). Relationship between Foot Length Measurements and Body Height: A Prospective Regional Study among Adolescents in Central Region of Kosovo. Journal of Contemporary Medical Sciences, in press

Masanovic, B., Gardasevic, J., \& Arifi, F. (2018d). Standing Height and its Estimation Utilizing Foot Length Measurements in Adolescents from Southern Region in Kosovo. Sport Mont, 16(2), 101-6.

Masanovic, B., Gardasevic, J., \& Arifi, F. (2018e). Sitting Height/Standing Height Relationship in Southern Region of Kosovo. Retrieved from SSRN's eLibrary: https://ssrn.com/abstract=3138523

Masanovic, B., Gardasevic, J., \& Arifi, F. (2018f). Sitting Height/Standing Height Relations in Central Region of Kosovo. Retrieved from SSRN's eLibrary: https://ssrn.com/abstract=3138525

Masanovic, B., Gardasevic, J., \& Arifi, F. (2018g). Sitting Height/Standing Height Relationship Measurements in Northern Region of Kosovo. Retrieved from SSRN's eLibrary: https://ssrn.com/abstract=3138526

Milašinović, R., Gardašević, J., \& Bjelica, D. (2017). Body heiht and its estimation utilizing arm span measurements in male adolescents from northern region in Montenegro. Acta Kinesiologica. (2) 75-80.

Milasinovic, R., Popovic, S., Jaksic, D., Vasiljevic, I. \& Bjelica, D. (2016b). Stature and its Estimation Utilizing Arm Span Measurements in Feale Adole- scents from Southern Region in Montenegro. Sport Mont, 14(3), 15-18. Milasinovic, R., Popovic, S., Matic, R., Gardasevic, J. \& Bjelica, D. (2016a). Body Height and its Estimation Utilizing Arm Span Measurements in Male Adolescents from Southern Region in Montenegro. Sport Mont, 14(2), 21-3.

Mohanty, S.P., Babu, S.S. \& Nair, N.S., (2001).The use of arm span as a predictor of height. A study of South Indian women. Journal of Orthopedics Surgery 9(1), 19-23.

Pineau, J. C., Delamarche, P., \& Bozinovic, S. (2005). Average height of adolescents in the Dinaric Alps (in French). Comptes Rendus Biologies, 328(9), 841-6.

Popovic, S. \& Bjelica, D. (2016). Body Height and its Estimation Utilizing Arm Span Measurements in Kosovan Adolescence: National Survey. In Abstract Book of International Eurasian Conference on Sport, Education, and Society (9), Antalya: International Science Culture and Sport Association.

Popovic, S. (2016). Body Height and its Estimation Utilizing Arm Span Measurements in Montenegrin Adults: National Survey. In Book of Summaries of 11th FIEP European Congress "Anthropological Aspects of Sport, Physical Education and Recreation" (5-6), Banjaluka: University of Banjaluka, Faculty of Physical Education and Sport.

Popovic, S. (2017). Local Geographical Differences in Adult Body Height in Montenegro. Montenegrin Journal of Sports Science and Medicine, 6(1), 81-87.

Popovic, S. Gardasevic, J., Masanovic, B., Arifi, F., \& Bjelica, D. (2017b). Standing Height and its Estimation Utilizing Foot Length Measurements in Adolescents from Western Region in Kosovo. Sport Mont, 15(3), 3-7.

Popovic, S., \& Bjelica, D. (2016). Body Height and its Estimation Utilizing Arm Span Measurements in Kosovan Adolescence: National Survey. In Abstract Book of International Eurasian Conference on Sport, Education, and Society (9), Antalya: International Science Culture and Sport Association.

Popovic, S., \& Bjelica, D. (2017). Body Height and its Estimation Utilizing Foot Length Measurements in Kosovan Adults: National Survey. In Abstract Book of the Sport Science Conference AESA 2017 (2), Amol: Faculty of Sport Sciences, Shomal University; Asian Exercise and Sport Science Association.

Popovic, S., Arifi, F., \& Bjelica, D. (2017a). Standing Height and its Estimation Utilizing Foot Length Measurements in Kosovan Adults: National Survey. International Journal of Applied Exercise Physiology, 6(2), 1-7.

Popovic, S., Bjelica, D., \& Hadzic, R. (2014a). Average body height of adolescents in Montenegro. In Proceedings book of the 13th International Sport Sciences Congress (462-463). Konya: Selcuk University.

Popovic, S., Bjelica, D., Georgiev, G., Krivokapic, D., \& Milasinovic, R. (2016). Body Height and its Estimation Utilizing Arm Span Measurements in Macedonian Adults. Anthropologist, 24(3), 737-745.

Popovic, S., Bjelica, D., Jaksic, D. \& Hadzic, R. (2014b). Comparative Study of Anthropometric Measurement and Body Composition between Elite Soccer and Volleyball Players. International Journal of Morphology, 32(1), 267- 74.

Popović, S., Bjelica, D., Milašinović, R., \& Gardašević, J. (2016). Body height and its estimation utilizing arm span measurements in male adolescents from northern region in Montenegro. In Book of Abstracts of 4th International Scientific Conference "Exercise and Quality of Life" (38), Novi Sad: Faculty of Sport and Physical Education.

Popović, S., Bjelica, D., Milašinović, R., Gardašević, J., \& Rašidagić, F. (2016). Body height and its estimation utilizing arm span measurements in male adolescents from Herzeg-Bosnia entity in Bosnia and Herzegovina. In Book of Abstracts of IUAES Inter Congress "World anthropologies and privatization of knowledge: engaging anthropology in public" (148), Dubrovnik: International Union of Anthropological and Ethnological Sciences.

Popovic, S., Bjelica, D., Molnar, S., Jaksic, D., \& Akpinar, S. (2013). Body Height and Its Estimation Utilizing Arm Span Measurements in Serbian Adults. International Journal of Morphology, 31(1), 271-9.

Popovic, S., Bjelica, D., Molnar, S., Jaksic, D., \& Akpinar, S. (2013). Body Height and Its Estimation Utilizing Arm Span Measurements in Serbian Adults. International Journal of Morphology, 31(1), 271-279.

Popovic, S., Bjelica, D., Petkovic, J.\& Muratovic, A. (2012). Comparative Study of Anthropometric Measurement and Body Composition between Elite Soccer and Handball Players. In Proceedings Book of the 4th International Scientific Conference "Contemporary Kinesiology"(102-108). Split: Faculty of Kinesiology, University of Split.

Popovic, S., Bjelica, D., Tanase, G.D., \& Milasinovic, R. (2015). Body Height and Its Estimation Utilizing Arm Span Measurements in Bosnian and Herzegovinian Adults. Montenegrin Journal of Sports Science and Medicine, 4(1), 29-36.

Popović, S., Milašinović, R., Jakšić, D., Vasiljević, I., Bjelica, D. (2016). Body 
height and its estimation utilizing arm span measurements in female adolescents from southern region in Montenegro. In Book of Abstracts of the 13th International Scientific Conference on Transformation Proces in Sport "Sport Performance" (30), Podgorica: Montenegrin Sports Academy.

Popović, S., Milašinović, R., Matić, R., Gardašević, J., Bjelica, D. (2016). Body height and its estimation utilizing arm span measurements in male adolescents from southern region in Montenegro. In Book of Abstracts of the 13th International Scientific Conference on Transformation Process in Sport "Sport Performance" (29-30), Podgorica: Montenegrin Sports Academy.

Quanjer, P.H., Capderou, A., Mazocioglu, M.M., Aggarwal, A., Popovic, S.
Datta Banik, S., Tayie, F.A. K., Golshan, M., Ip, M.S.M., \& Zelter, M. (2014) All-age relationship between arm span and height in different ethnic groups. European Respiratory Journal, 44, 905-12.

Schönbeck, Y., Talma, H., Van Dommelen, P., Bakker, B., Buitendijk, S., Hirasing, R., \& Van Buuren, S. (2013). The world's tallest nation has stopped growing taller: the height of Dutch children from 1955 to 2009. Pediatric Research, 73(3), 371-7.

Ter Goon, D., Toriola, A.T., Musa, D.I., \& Akusu, S. (2011). The relationship between a span and Body Height in Nigerian adults. Kinesiology, 43(1), 38-43.

Vujovic, D., Bubanja, M., Tanase, G. D. \& Milasinovic, R. (2015). Body Height and Its Estimation Utilizing Arm Span Measurements in Male Adolescents from Central Region in Montenegro. Sport Mont, 12(43-45), 283-8. 